University of Wollongong

Research Online

Faculty of Informatics - Papers (Archive)

Faculty of Engineering and Information

Sciences

$1-1-2007$

\title{
Modeling and solving Semiring Constraint Satisfaction Problems by transformation to Weighted Semiring Max-SAT
}

Louise Leenen

University of Wollongong, I1916@uow.edu.au

Anbulagan Anbulagan

Australian National University

Thomas Meyer

Meraka Institute, South Africa

Aditya K. Ghose

University of Wollongong, aditya@uow.edu.au

Follow this and additional works at: https://ro.uow.edu.au/infopapers

Part of the Physical Sciences and Mathematics Commons

\section{Recommended Citation}

Leenen, Louise; Anbulagan, Anbulagan; Meyer, Thomas; and Ghose, Aditya K.: Modeling and solving Semiring Constraint Satisfaction Problems by transformation to Weighted Semiring Max-SAT 2007, 202-212.

https://ro.uow.edu.au/infopapers/1394

Research Online is the open access institutional repository for the University of Wollongong. For further information contact the UOW Library: research-pubs@uow.edu.au 


\title{
Modeling and solving Semiring Constraint Satisfaction Problems by transformation to Weighted Semiring Max-SAT
}

\begin{abstract}
We present a variant of the Weighted Maximum Satisfiability Problem(Weighted Max-SAT), which is a modeling of the Semiring Con- straint Satisfaction framework. We show how to encode a Semiring Constraint Satisfaction Problem (SCSP) into an instance of a propositional Weighted Max-SAT, and call the encoding Weighted Semiring Max-SAT (WS-Max-SAT). The clauses in our encoding are highly structured and we exploit this feature to develop two algorithms for solving WS-Max-SAT: an incomplete algorithm based on the well-known GSAT algorithm for Max-SAT, and a branch-and-bound algorithm which is complete. Our preliminary experiments show that the translation of SCSP into WS- Max-SAT is feasible, and that our branch-and-bound algorithm performs surprisingly well. We aim in future to combine the natural exible rep- resentation of the SCSP framework with the inherent efficiencies of SAT solvers by adjusting existing SAT solvers to solve WS-Max-SAT.
\end{abstract}

\section{Keywords}

Modeling, solving, Semiring, Constraint, Satisfaction, Problems, transformation, Weighted, Semiring, Max, SAT

\section{Disciplines}

Physical Sciences and Mathematics

\section{Publication Details}

Leenen, L., Anbulagan, A., Meyer, T. \& Ghose, A. K. (2007). Modeling and solving Semiring Constraint Satisfaction Problems by transformation to Weighted Semiring Max-SAT. In M. Orgun \& J. Thornton (Eds.), Al 2007: Advances in Artificial Intelligence (pp. 202-212). Berlin: Springer-Verlag. 


\title{
Modeling and Solving Semiring Constraint Satisfaction Problems by Transformation to Weighted Semiring Max-SAT
}

\author{
Louise Leenen ${ }^{1}$, Anbulagan $^{2}$, Thomas Meyer ${ }^{3}$, and Aditya Ghose ${ }^{4}$ \\ 1 DSL, SCSSE, University of Wollongong, Australia and DPSS, CSIR, South Africa \\ 2 NICTA $^{\star \star}$ and ANU, Canberra, Australia \\ ${ }^{3}$ Meraka Institute, CSIR, Pretoria, South Africa \\ 4 DSL, SCSSE, University of Wollongong, Australia
}

\begin{abstract}
We present a variant of the Weighted Maximum Satisfiability Problem (Weighted Max-SAT), which is a modeling of the Semiring Constraint Satisfaction framework. We show how to encode a Semiring Constraint Satisfaction Problem (SCSP) into an instance of a propositional Weighted Max-SAT, and call the encoding Weighted Semiring Max-SAT (WS-Max-SAT). The clauses in our encoding are highly structured and we exploit this feature to develop two algorithms for solving WS-MaxSAT: an incomplete algorithm based on the well-known GSAT algorithm for Max-SAT, and a branch-and-bound algorithm which is complete. Our preliminary experiments show that the translation of SCSP into WSMax-SAT is feasible, and that our branch-and-bound algorithm performs surprisingly well. We aim in future to combine the natural flexible representation of the SCSP framework with the inherent efficiencies of SAT solvers by adjusting existing SAT solvers to solve WS-Max-SAT.
\end{abstract}

\section{Introduction}

The Semiring Constraint Satisfaction Problem (SCSP) framework is an approach to constraint satisfaction and optimization that generalises classical Constraint Satisfaction Problems (CSPs), Partial Constraint Satisfaction Problems, Fuzzy CSPs, Weighted CSPs, Probabilistic CSPs, and others (over finite domains) [14], while also permitting the specification of interesting new instances. Considerable interest has been shown in solving SCSPs. Complete methods exist [5-7] but are likely to require exponential time. Bistarelli et al. [8] present a prototype SCSP solver based on an incomplete local search method. Wilson [9] shows how to use decision diagrams to solve SCSPs but provides no implementation.

A significant amount of work has been devoted to solving propositional satisfiability (SAT) problems, and specifically the well-known maximum satisfiability problem (Max-SAT) $[10,11]$. There is continuing interest in translations between CSPs and SAT problems [12-14]. This prompted us to explore the application of methods for solving Max-SAT to SCSPs. We modify the support encoding of CSP to SAT by Gent [12] in order to translate SCSPs as a variant of the Weighted

** NICTA is funded through the Australian Government's Backing Australia's Ability initiative, in part through the Australian Research Council. 
Max-SAT Problem, and call this encoding WS-Max-SAT. Our encoding results in propositional clauses whose structure can be exploited in our algorithms to solve WS-Max-SAT: a local search algorithm that is a modification of the GSAT algorithm for solving Max-SAT [15], and a branch-and-bound (BnB) algorithm.

We show how to formulate a SCSP as a WS-Max-SAT, present a local search and a BnB algorithm, and some experimental results. A comparison of our BnB algorithm with CONFLEX [3], a fuzzy CSP solver, shows that our algorithm performs surprisingly well, and is significantly faster than CONFLEX.

\section{Semiring Constraint Satisfaction Problems}

The SCSP framework is a popular approach for the representation of constraint satisfaction and in particular, partial constraint satisfaction problems [1].

Definition 1. A c-semiring is a tuple $S=\langle A,+, \times, \mathbf{0}, \mathbf{1}\rangle$ such that i) $A$ is a set with $\mathbf{0}, \mathbf{1} \in A$;

ii) + is defined over (possibly infinite) sets of elements of $A$ as follows a) for all $a \in A, \sum(\{a\})=a$; b) $\sum(\emptyset)=\mathbf{0}$ and $\sum(A)=\mathbf{1}$; c) $\sum\left(\bigcup A_{i}, i \in I\right)=$ $\sum\left(\left\{\sum\left(A_{i}\right), i \in I\right\}\right)$ for all sets of indices I (flattening property);

iii) $\times$ is a commutative, associative, and binary operation with $\mathbf{1}$ as unit element and $\mathbf{0}$ as absorbing element; iv) $\times$ distributes over + (i.e., for any $a \in A$ and $\left.B \subseteq A, a \times \sum(B)=\sum(\{a \times b, b \in B\})\right)$.

The elements of the set $A$ are the preference values to be assigned to tuples of values of the domains of constraints. A partial ordering $\leqslant_{S}$ over the set $A$ can be defined: $\alpha \leqslant_{S} \beta$ iff $\alpha+\beta=\beta$.

Definition 2. A constraint system is a 3-tuple $C S=\left\langle S_{p}, D, V\right\rangle$, where $S_{p}=$ $\left\langle A_{p},+_{p}, \times_{p}, \mathbf{0}, \mathbf{1}\right\rangle$ is a c-semiring, $V$ is an ordered finite set of variables, and $D$ is a finite set containing the allowed values for the variables in $V$. Given $C S=\left\langle S_{p}, D, V\right\rangle$, where $S_{p}=\left\langle A_{p},+_{p}, \times_{p}, \mathbf{0}, \mathbf{1}\right\rangle$, a constraint over $C S$ is a pair $c=\left\langle d e f_{c}^{p}\right.$, con $\left._{c}\right\rangle$ where con ${ }_{c} \subseteq V$ is called the signature of the constraint, and de $f_{c}^{p}: D^{k} \rightarrow A_{p}$ ( $k$ is the cardinality of con $_{c}$ ) is called the value of the constraint.

Definition 3. Given $C S=\left\langle S_{p}, D, V\right\rangle$, a SCSP over $C S$ is a pair $P=\langle C$, con $\rangle$ where $C$ is a finite set of constraints over $C S$ and con $=\bigcup_{c \in C}$ con $_{c}$. Assume $\left\langle d e f_{c_{1}}^{p}\right.$, con $\left._{c}\right\rangle \in C$ and $\left\langle\operatorname{def}_{c_{2}}^{p}\right.$, con $\left._{c}\right\rangle \in C$ implies de $f_{c_{1}}^{p}=\operatorname{def}_{c_{2}}^{p}$.

Definition 4. Given $C S=\left\langle S_{p}, D, V\right\rangle$ with $V$ totally ordered via $\preceq$, consider any $k$-tuple $t=\left\langle t_{1}, t_{2}, \ldots, t_{k}\right\rangle$ of values of $D$ and two sets $W=\left\{w_{1}, \ldots, w_{k}\right\}$ and $W^{\prime}=\left\{w_{1}^{\prime}, \ldots, w_{m}^{\prime}\right\}$ such that $W^{\prime} \subseteq W \subseteq V$ and $w_{i} \preceq w_{j}$ if $i \leq j$ and $w_{i}^{\prime}$ $\preceq w_{j}^{\prime}$ if $i \leq j$. Then the projection of $t$ from $W$ to $W^{\prime}$, written $t \downarrow_{W^{\prime}}$, is defined as the tuple $t^{\prime}=\left\langle t_{1}^{\prime}, \ldots, t_{m}^{\prime}\right\rangle$ with $t_{i}^{\prime}=t_{j}$ iff $w_{i}^{\prime}=w_{j}$.

For $C S=\left\langle S_{p}, D, V\right\rangle, S_{p}=\left\langle A_{p},+_{p}, \times_{p}, \mathbf{0}, \mathbf{1}\right\rangle$, and two constraints $c_{1}=\left\langle\operatorname{def}_{c_{1}}^{p}\right.$, con $\left._{c_{1}}\right\rangle$ and $c_{2}=\left\langle\right.$ def $_{c_{2}}^{p}$, con $\left._{c_{2}}\right\rangle$ over $C S$, their combination, $c_{1} \otimes c_{2}$, is the constraint $c=\left\langle\operatorname{def}_{c}^{p}, \operatorname{con}_{c}\right\rangle$ with $\operatorname{con}_{c}=\operatorname{con}_{c_{1}} \cup \operatorname{con}_{c_{2}}$ and $\operatorname{def}_{c}^{p}(t)=\operatorname{def}_{c_{1}}^{p}\left(t \downarrow_{\operatorname{con}_{c_{1}}}^{\operatorname{con}_{c}}\right) \times_{p}$ $\operatorname{def} f_{c_{2}}^{p}\left(t \downarrow_{\text {con }_{c_{2}}}^{\text {con }_{c}}\right)$. Let $\otimes C$ denote $c_{1} \otimes \ldots \otimes c_{m}$ with $C=\left\{c_{1}, \ldots, c_{m}\right\}$. 
Definition 5. Given $C S=\left\langle S_{p}, D, V\right\rangle$, with $S_{p}=\left\langle A_{p},+_{p}, \times_{p}, \mathbf{0}, \mathbf{1}\right\rangle$, a constraint $c=\left\langle d e f_{c}^{p}\right.$, con $\left._{c}\right\rangle$ over $C S$, and a set I of variables $(I \subseteq V)$, the projection of $c$ over I, written $c \Downarrow I$, is the constraint $c^{\prime}=\left\langle d e f_{c^{\prime}}^{p}\right.$, con $\left._{c^{\prime}}\right\rangle$ over CS with $\operatorname{con}_{c^{\prime}}=I \cap \operatorname{con}_{c}$ and $\operatorname{de}_{c^{\prime}}^{p}\left(t^{\prime}\right)=\sum_{\left\{t \mid t \downarrow_{I I n o n_{c}}^{c o n n_{c}}=t^{\prime}\right\}} \operatorname{def}_{c}^{p}(t)$. Given a SCSP P = $\langle C$, con $\rangle$ over $C S$, the solution of $P$ is a constraint defined as $\operatorname{Sol}(P)=\otimes C$.

Definition 6. Given a SCSP problem $P=\langle C$, con $\rangle$, consider $\operatorname{Sol}(P)=\left\langle\operatorname{de} f_{c}^{p}\right.$, con $\rangle$. The abstract solution of $P$ is the set $A \operatorname{Sol}(P)=\left\{\langle t, v\rangle \mid \operatorname{de} f_{c}^{p}(t)=v\right.$ and there is no $t^{\prime}$ such that $\left.v<_{S_{p}} \operatorname{de} f_{c}^{p}\left(t^{\prime}\right)\right\}$. Let $A \operatorname{Sol} V(P)=\{v \mid\langle t, v\rangle \in A \operatorname{Sol}(P)\}$.

\section{Max-SAT and Weighted Max-SAT}

The SAT problem is to determine whether a given propositional formula has a model (is satisfiable) or is unsatisfiable. The maximum satisfiability problem is the optimisation variant of SAT: given a set of clauses, the problem is to find a variable assignment that maximises the number of satisfied clauses. In Weighted Max-SAT a weight is assigned to each clause and the goal becomes to maximise the total weight of the satisfied clauses. More formally, let $C=\left\{C_{1}, C_{2}, \ldots, C_{m}\right\}$ be the set of clauses that involve $n$ Boolean variables $x_{1}, x_{2}, \ldots, x_{n}$ that can take the value true or false, and $w_{i}$ with $i=1, \ldots, m$, is the weight assigned to each clause. The objective function is defined as $f(x)=\sum_{i=1}^{m} w_{i} \cdot I\left(C_{i}\right)$, where $I\left(C_{i}\right)$ is one if and only if the clause $C_{i}$ is satisfied, and otherwise is zero. When we refer to Max-SAT in the remainder of this paper we assume the weighted version.

Some of the best exact Max-SAT solvers $[16,10,11,17,18]$ implement variants of a $\mathrm{BnB}$ algorithm: given a $\mathrm{CNF}$ formula $\theta$, a $\mathrm{BnB}$ algorithm explores the search tree representing all possible assignments for $\theta$ in a depth-first manner. The lower bound (LB) of a node is the sum of the weights of all clauses satisfied by the current partial assignment plus an over-estimation of the sum of the weigths of all clauses that will become satisfied if the current assignment is completed. If $\mathrm{LB} \leq \mathrm{UB}$ (UB is the upper bound, or best solution found so far), the subtree below the current node is pruned, and the algorithm backtracks to a node at a higher level in the tree. If LB > UB, the algorithm tries to find a better solution by extending the current partial solution by instantiating one more variable. The solution is the value of UB after the whole tree has been explored.

\section{Weighted Semiring Max-SAT}

In this section we show how to encode a SCSP into a WS-Max-SAT. Our encoding of a SCSP into a WS-Max-SAT is based on the support encoding of CSPs into Boolean SAT [12]. Every propositional clause in our encoding will receive a semiring value from the semiring associated with the SCSP as a weight.

For the remainder of this paper let $P=\langle C$, con $\rangle$ be a SCSP over a constraint system $C S=\left\langle S_{p}, D, V\right\rangle$, such that every variable $i \in V$ has $D=\left\{d_{1}, \ldots, d_{d}\right\}$ as its domain, and $S_{p}=\left\langle A_{p},+_{p}, \times_{p}, \mathbf{0}, \mathbf{1}\right\rangle$. Assume the cardinality of $V$ is $n$, the cardinality of $C$ is $m$, and that there are $s$ semiring values in the set $A_{p}$. Every constraint $c \in C, c=\left\langle d e f_{c}^{p}\right.$, con $\rangle$ with $r$ the cardinality of $\operatorname{con}_{c}$ and $\operatorname{con}_{c} \subseteq V$.

For every SCSP variable $i \in V$, we have $d$ WS-Max-SAT variables: the WSMax-SAT variable $X_{i, j}$ is true if the SCSP variable $i$ has the value $d_{j} \in D$ with 
$j=1 \ldots d$. Our WS-Max-SAT encoding (encoding of $P$ ) has three kinds of clauses:

1. at-least-one clauses to ensure that each SCSP variable is assigned at least one value from its domain;

2. at-most-one clauses to ensure that each SCSP variable is assigned at most one value from its domain;

3. support clauses to represent all the possible value pairs (tuples) with their associated semiring values for every constraint. For every constraint we have at most $s$ such clauses, and every clause receives the associated semiring value as its weight.

- For every variable $i \in V$ we have one at-least-one clause: $X_{i, 1} \vee X_{i, 2} \vee \ldots \vee X_{i, d}$.

- For every variable $i \in V$ we have $0.5[d(d-1)]$ at-most-one clauses: one clause, $\neg X_{i, v} \vee \neg X_{i, w}$, for every pair $\left(d_{v}, d_{w}\right)$, where $1 \leq v<w \leq d$.

- For every constraint, we have at most $s$ support clauses: one clause to represent all the tuples that have the same associated semiring value. Suppose $A_{p}=\left\{p_{1}, p_{2}, \ldots, p_{s}\right\}$, then every constraint $c \in C$ has at most $s$ support clauses, $\sup _{c}^{p_{t}}$ with $t=1, \ldots, s$. A support clause $\sup _{c}^{p_{t}}$ for a constraint $c=\left\langle\operatorname{def}_{c}^{p}, \operatorname{con}_{c}\right\rangle$ is a disjunction of the representation of all tuples $\left\langle X_{c_{1}, v_{1}}, \ldots, X_{c_{r}, v_{r}}\right\rangle$ (the conjunction of all these literals) that has the associated semiring value $p_{t}, c_{i} \in \operatorname{con}_{c}$, and $v_{i} \in D$ for $i=1 \ldots r$.

All the clauses except the support clauses have to be satisfied and we call these clauses the hard clauses. The minimum semiring value, $\mathbf{0}$, is assigned as the weight of the negation of each hard clause. If a hard clause is not satisfied by an assignment, this unsatisfied clause contributes the minimum semiring value to the total weight. Note that at most one support clause for each constraint can be satisfied by a truth assignment if we assume that the at-most-one and atleast-one clauses are all satisfied. We regard the support clauses as soft clauses: the weight assigned to each support clause is the combined semiring value of the tuples represented in that support clause.

Definition 7. Given a SCSP P and a WS-Max-SAT encoding of P, let the set Sup contain the support clauses in the encoding and $\alpha$ be some truth assignment for the clauses in Sup. Then $t_{\text {sol }}^{\alpha}=\left\langle X_{1, v_{a l}}, \ldots, X_{n, v_{1} l_{n}}\right\rangle$ is a solution tuple for the encoding iff the following holds:

- for every constraint $c \in C$ exactly one of its support clauses, sup $p_{c}^{k},(1 \leq k \leq$ $\left.p_{s}\right)$ is satisfied by $\alpha$. This support clause represents a disjunction of tuples of this constraint, each as a conjunction of WS-Max-SAT variables, with exactly one tuple, $t_{c}=\left\langle X_{c_{1}}, \ldots, X_{c_{r}}\right\rangle$ with $c_{i} \in \operatorname{con}_{c}, v_{i} \in D, i=1 \ldots r$, and such that all $X_{c_{i}, v_{i}}=$ true, and

- if $c_{i}$ is equal to $j(j=1, \ldots, n)$ then $v_{i}=v_{a l}$.

The semiring value associated with $t_{\text {sol }}^{\alpha}$ is $s r_{\text {sol }}^{\alpha}=s p_{1} \times_{p} \ldots \times_{p} s p_{m}$ where $s p_{c}=k$ and $\sup _{c}^{k}$ is the support clause satisfied by assignment $\alpha$ for a constraint $c$.

Definition 8. Given a SCSP $P$, let $C l$ be the set of clauses that represents the WS-Max-SAT encoding of P. Let $C l_{h}=\left\langle c l_{1}, \ldots c l_{h}\right\} \subseteq C l$ be the clauses 
that are satisfied by some truth assignment $\alpha$. The WS-Max-SAT problem is to find $\max f(\alpha)=\bigotimes_{i=1}^{h} w_{i}$, where $w_{i} \in A_{p}$ is the weight assigned to clause $i \in C l_{h}, f(\alpha)$ is maximal with respect to the partial order $<_{S_{p}}$, and $t_{\text {sol }}^{\alpha}=$ $\left\langle X_{1, v_{a l}}, \ldots, X_{n, v_{a l}}\right\rangle$ is the solution tuple for the encoding $C l$. The ordered pair $\left\langle\left\langle\right.\right.$ val $_{1}, \ldots$, val $\left.\left._{n}\right\rangle, f(\alpha)\right\rangle \in A \operatorname{Sol}(P)$.

Example 1. Suppose we have a SCSP $P=\left\langle\left\{c_{1}, c_{2}, c_{3}\right\}, V\right\rangle$ over $\left\langle S_{p}, D, V\right\rangle$, with $V=\{A, B, C\}$ and $D=\{1,2,3\}$. The three constraints are explicitly defined by $c_{1}: A<B, c_{2}: B<C$, and $c_{3}: C<A$. The semiring is $S_{p}=\langle\{0,5,10\}, \max , \min , 0,10\rangle$. See the constraint definitions in Table 1 . Note

\begin{tabular}{|c|c|c|c|}
\hline $\mathrm{t}$ & $c_{1}: A<B$ & $c_{2}: B<C$ & $c_{3}: C<A$ \\
\hline$\langle 1,1\rangle$ & 5 & 5 & 5 \\
\hline$\langle 1,2\rangle$ & 10 & 10 & 10 \\
\hline$\langle 1,3\rangle$ & 10 & 10 & 10 \\
\hline$\langle 2,1\rangle$ & 0 & 0 & 0 \\
\hline$\langle 2,2\rangle$ & 5 & 5 & 5 \\
\hline
\end{tabular}

\begin{tabular}{|c|c|c|c|}
\hline $\mathrm{t}$ & $c_{1}: A<B$ & $c_{2}: B<C$ & $c_{3}: C<A$ \\
\hline$\langle 2,3\rangle$ & 10 & 10 & 10 \\
\hline$\langle 3,1\rangle$ & 0 & 0 & 0 \\
\hline$\langle 3,2\rangle$ & 0 & 0 & 0 \\
\hline$\langle 3,3\rangle$ & 5 & 5 & 5 \\
\hline
\end{tabular}

Table 1. Constraint definitions

that the first coordinate reflects the value of the first variable in a constraint and the second coordinate reflects the value of the second variable. For example, the preference value associated with the tuple $\langle 1,3\rangle$ for constraint $c_{1}$ is when $A$ has the value 1 and $B$ has the value 3 . Tuples that satisfy the constraints get the highest preference value. Among the tuples that do not satisfy the constraints, we prefer those where the first coordinate equals the second coordinate. These tuples get a preference value of 5, and all the remaining tuples get the worst value, 0 . The WS-Max-SAT encoding of the problem follows below.

At-least-one clauses: $A_{1} \vee A_{2} \vee A_{3}, B_{1} \vee B_{2} \vee B_{3}$, and $C_{1} \vee C_{2} \vee C_{3}$

At-most-one clauses: $\neg A_{1} \vee \neg A_{2}, \neg A_{2} \vee \neg A_{3}, \neg A_{1} \vee \neg A_{3}, \neg B_{1} \vee \neg B_{2}, \neg B_{2} \vee \neg B_{3}$, $\neg B_{1} \vee \neg B_{3}, \neg C_{1} \vee \neg C_{2}, \neg C_{2} \vee \neg C_{3}$, and $\neg C_{1} \vee \neg C_{3}$

Support clauses:

$c_{1}^{10}:\left[\left(A_{1} \wedge B_{2}\right) \vee\left(A_{1} \wedge B_{3}\right) \vee\left(A_{2} \wedge B_{3}\right)\right], c_{1}^{5}:\left[\left(A_{1} \wedge B_{1}\right) \vee\left(A_{2} \wedge B_{2}\right) \vee\left(A_{3} \wedge B_{3}\right)\right]$,

$c_{1}^{0}:\left[\left(A_{2} \wedge B_{1}\right) \vee\left(A_{3} \wedge B_{1}\right) \vee\left(A_{3} \wedge B_{2}\right)\right], c_{2}^{10}:\left[\left(B_{1} \wedge C_{2}\right) \vee\left(B_{1} \wedge C_{3}\right) \vee\left(B_{2} \wedge C_{3}\right)\right]$,

$c_{2}^{5}:\left[\left(B_{1} \wedge C_{1}\right) \vee\left(B_{2} \wedge C_{2}\right) \vee\left(B_{3} \wedge C_{3}\right)\right], c_{2}^{0}:\left[\left(B_{2} \wedge C_{1}\right) \vee\left(B_{3} \wedge C_{1}\right) \vee\left(B_{3} \wedge C_{2}\right)\right]$,

$c_{3}^{10}:\left[\left(C_{1} \wedge A_{2}\right) \vee\left(C_{1} \wedge A_{3}\right) \vee\left(C_{2} \wedge A_{3}\right)\right], c_{3}^{5}:\left[\left(C_{1} \wedge A_{1}\right) \vee\left(C_{2} \wedge A_{2}\right) \vee\left(C_{3} \wedge A_{3}\right)\right]$, $c_{3}^{0}:\left[\left(C_{2} \wedge A_{1}\right) \vee\left(C_{3} \wedge A_{1}\right) \vee\left(C_{3} \wedge A_{2}\right)\right]$

In our example, each support clause has its associated semiring value as its weight. The negation of all the remaining clauses are each given a weight of 0 :
1. $\neg A_{1} \wedge \neg A_{2} \wedge \neg A_{3}$
2. $\neg B_{1} \wedge \neg B_{2} \wedge \neg B_{3}$
3. $\neg C_{1} \wedge \neg C_{2} \wedge \neg C_{3}$
4. $A_{1} \wedge A_{2}$
5. $A_{2} \wedge A_{3}$
6. $A_{1} \wedge A_{3}$
7. $B_{1} \wedge B_{2}$
8. $B_{2} \wedge B_{3}$
9. $B_{1} \wedge B_{3}$
10. $C_{1} \wedge C_{2}$
11. $C_{2} \wedge C_{3}$
12. $C_{1} \wedge C_{3}$

We want to find an assignment that does not satisfy any of the (hard) clauses numbered 1 to 12 . A truth assignment that satisfies one or more of the support clauses with an associated semiring value of 10 is preferred over one with lower semiring values. We can prove that our encoding is correct. 
Theorem 1. Let SCSP $P$ be a set of clauses $C_{l}$ containing a WS-Max-SAT encoding for $P$, and a truth assignment $\alpha$.

- If $t_{\text {sol }}^{\alpha}=\left\langle X_{1, v_{1} l_{1}}, \ldots, X_{n, v a l_{n}}\right\rangle$ is a solution tuple for the WS-Max-SAT encoding of $P$, then $t=\left\langle X_{1}, \ldots, X_{n}\right\rangle$ with $X_{i}=$ val $_{i}$ for $i=1, \ldots, n$ is a solution tuple for $P$.

- If sr ${ }_{\text {sol }}^{\alpha}$ is the semiring value associated with $t_{\text {sol }}^{\alpha}$, then the associated semiring value of $t$ is $s r_{\text {sol }}^{\alpha}$.

\section{Algorithms to solve WS-Max-SAT}

Most SAT and Max-SAT solvers require the propositional clauses to be in conjunctive normal form $(\mathrm{CNF})$. In our encoding, the propositional clauses are highly structured and we do not have to convert them into CNF. We only allow truth assignments where the at-least-one-variable and at-most-one-variable clauses are satisfied in our implementation. This leaves only the support clauses that have to be checked for satisfiability: exactly one support clause can be satisfied for every constraint. We simply search for the support clause with the best associated semiring value for each constraint under the current truth assignment.

\subsection{The GSAT Algorithm for WS-Max-SAT}

GSAT [15] is a greedy algorithm that tries to maximise the number of satisfied clauses by selecting different variable assignments based on the score of a variable $x$ under the current assignment $\alpha$ : this is defined as the difference between the weight of the clauses unsatisfied by $\alpha$ and the assignment obtained by flipping $x$ in $\alpha$. GSAT is not a complete algorithm and can get stuck in local minima. GSAT starts with a random truth assignment. After a maximum number of allowed flips has been performed, a new random truth assignment is generated. This improves the probability to find a solution.

We have adjusted GSAT, as presented in Algorithm 1, to solve a WS-MaxSAT problem for SCSPs with binary constraints. The procedure chooseVariable selects the next variable to be flipped by considering the score of each variable $x$ in WS-Max-SAT. The score of a variable $x$ in an assignment $\alpha$ is the $f$ value a truth assignment $\alpha_{x}$ has if $\alpha_{x}$ is identical to $\alpha$ except for the truth value assigned to $x$. There may be more than one variable with a maximum score. In this case any variable with a maximal $f$ value is chosen at random to be flipped. When a WS-Max-SAT variable $X_{i, j}$ is chosen to be flipped, we consider two cases:

- The current value of $X_{i, j}=$ true and has to be flipped to become false. Some variable $X_{i, l}$ with $l=1, \ldots, d$ and $l \neq j$ is chosen at random to become true.

- The current value of $X_{i, j}=$ false and has to be flipped to become true. Some variable $X_{i, l}$ with $l=1, \ldots, d$ and $l \neq j$ is currently true and is given the value false.

Example 2. Suppose we generate the following random truth assignment $\alpha_{1}$ : $A_{1}=A_{2}=B_{1}=B_{2}=C_{1}=C_{3}=$ false and $A_{3}=B_{3}=C_{2}=$ true. The following three clauses are satisfied: $c_{1}^{5}, c_{2}^{0}$, and $c_{3}^{10}$ with $f\left(\alpha_{1}\right)=\min (5,0,10)=$ 0 . The procedure chooseVariable finds that all flips result in $f$ values of 0 , so it randomly selects $C_{1}$ to be flipped. Now $C_{1}=$ true and $C_{2}=$ false. In the next iteration it finds that $C_{3}$ has a score of 5 and it flips $C_{3}$ : in this case the three clauses $c_{1}^{5}, c_{2}^{5}$, and $c_{3}^{5}$ with $f\left(\alpha_{3}\right)=\min (5,5,5)=5$. 


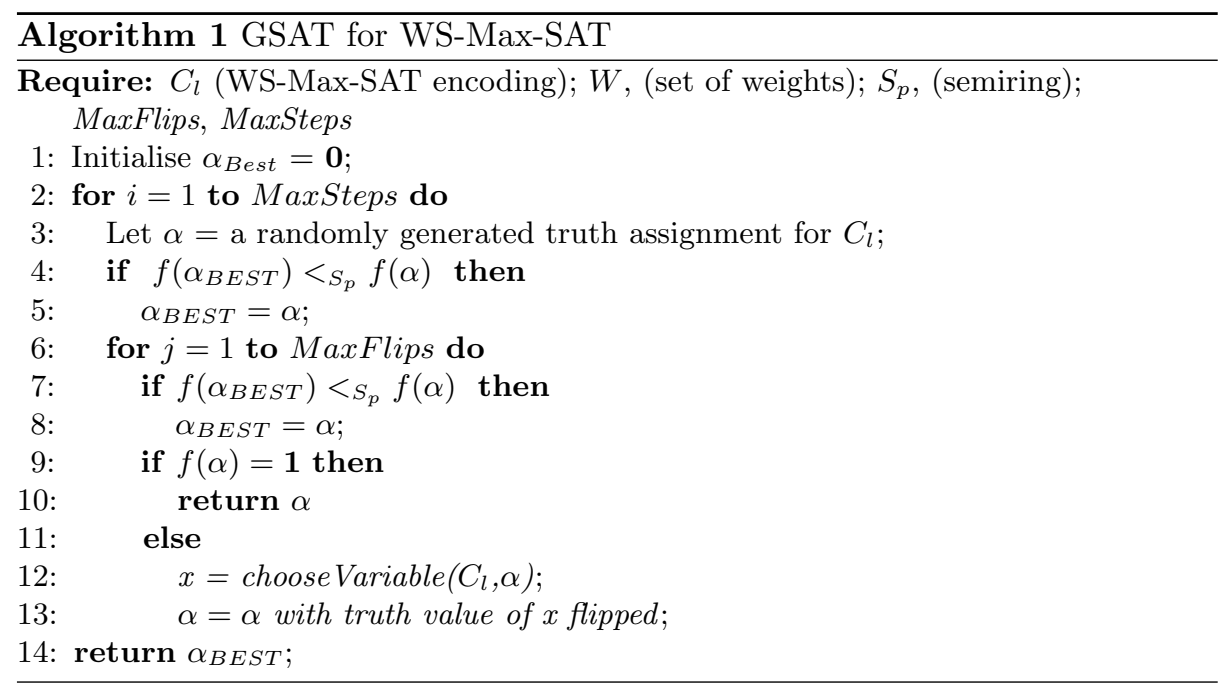

\subsection{The BnB Algorithm for WS-Max-SAT}

We present a BnB algorithm to solve WS-Max-SAT, as sketched in Algorithm 2. The variables are ordered according to their membership in the signatures of the constraints. We place the variables in the signature of the first constraint on a queue, and then check whether these variables appear in the signatures of other constraints. If they do, the unlisted variables in the others constraints' signatures are also placed on the queue. Repeat this step until all constraints' signatures have been checked. Every variable has a main constraint which is the constraint where the variable has been identified to be placed on the queue. To find a value for a variable we search among the tuples of its main constraint for a tuple with a maximal associated semiring value under the current partial assignment. From this chosen tuple we get a value to instantiate the current variable.

After a variable has been instantiated, we calculate a lower bound value for the current node in the search tree, i.e. we calculate an estimated semiring value for the variable-value tuple of the current (in most cases, partial) assignment. The lower bound is computed by looking at constraints whose signatures have been entirely instantiated by the current (possibly partial) solution and combining the semiring values assigned to the projection of the current solution to the signatures of these constraints. For all the remaining constraints (i.e with uninstantiated variables), we estimate the maximal semiring value for the purpose of finding a lower bound. The upper bound is initialised with the worst semiring value and the lower bound with the best semiring value. The variable var is instantiated with the next variable from Queue and var_domain contains the domain values for that variable.

\section{Experimental Setting and Results}

We used an Intel Pentium 4 processor at $2.53 \mathrm{GHz}$ with $512 \mathrm{MB}$ RAM. 


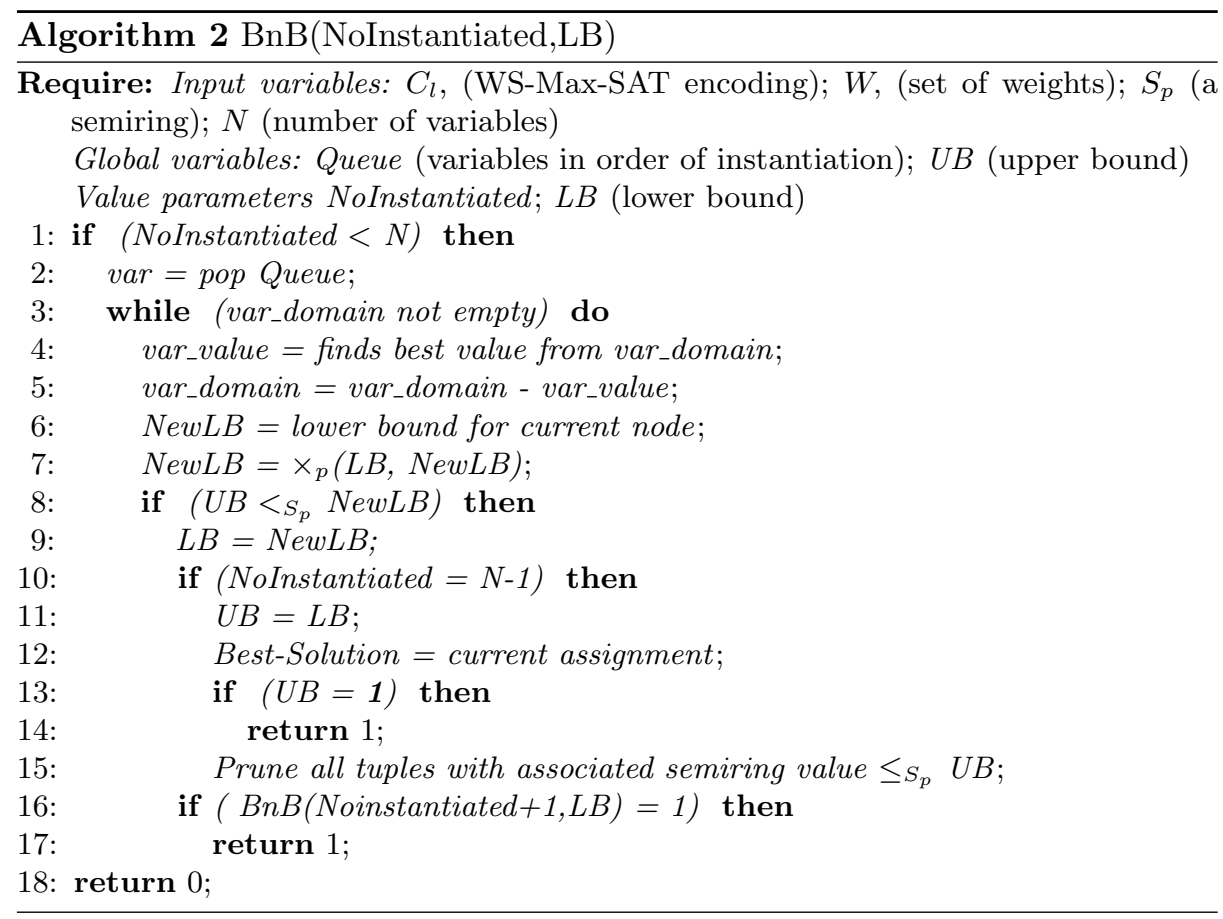

\subsection{Results of GSAT-based Algorithm}

We solved three sets of randomly generated binary fuzzy CSPs where each set contains 100 instances. Each instance has 10 domain values. Instances in Set 1 has 80 variables and 10 constraints, in Set 2100 variables and 10 constraints, and in Set 3 has 120 variables and 20 constraints.

In each set of 100 instances, 50 instances have a tightness of $70 \%$ and the other 50 instances have a tightness of $90 \%$. A tightness $(\mathrm{T})$ of $\mathrm{x} \%$ means that $(100-\mathrm{x}) \%$ of the possibles tuples have been assigned the maximum semiring value. All the problems have the following semiring values, $A_{p}=\{0,0.3,0.5,0.8,1\}$, the comparative operator is $\max$ and the combination operator is min. The results are in Table 2. In the third row $(S=1)$ we show for how many instances the algorithm found a maximal solution (with a solution tuple that has a semiring value of 1 ). In the next rows we show for how many instances we found solution tuples with semiring values of $0.8,0.5$ and 0.3 , respectively.

There were no instances where a solution with the minimum semiring value was found. The last two rows contain information for those instances where the maximal solution were found: it shows on average when the solution was found. We show during which step, and after how many flips, the algorithm halted. Each step consists of a maximum of 1000 flips.

All the problems instances have maximal solutions apart from three instances in set 3 with tightness $90 \%$ (last column). These three instances have a best 


\begin{tabular}{|c|c|c|c|c|c|c|}
\hline Set & 1 & 1 & 2 & 2 & 3 & 3 \\
\hline $\mathrm{T}$ & $70 \%$ & $90 \%$ & $70 \%$ & $90 \%$ & $70 \%$ & $90 \%$ \\
\hline $\mathrm{S}=1$ & 50 & 2 & 50 & 0 & 1 & 0 \\
\hline $\mathrm{S}=0.8$ & 0 & 48 & 0 & 49 & 33 & 0 \\
\hline $\mathrm{S}=0.5$ & 0 & 0 & 0 & 1 & 16 & 38 \\
\hline $\mathrm{S}=0.3$ & 0 & 0 & 0 & 0 & 0 & 12 \\
\hline \hline steps & 1.34 & 2.00 & 1.28 & n/a & 1 & n/a \\
\hline flips & 452.4 & 836.5 & 438.5 & n/a & 329.0 & n/a \\
\hline
\end{tabular}

Table 2. Results for the GSAT-based algorithm

solution with a combined semiring value of 0.8. Our GSAT algorithm performs reasonably well for a relatively small number of steps and flips.

\subsection{Results of BnB Algorithm}

We solved two sets of randomly generated binary fuzzy CSPs with CONFLEX [3] and with our BnB algorithm. We used the CONFLEX executable for Windows downloaded from the CONFLEX website. ${ }^{5}$ The 40 instances in Set 4 has 10 variables, 10 constraints, 10 domain values (20 instances with tightness $60 \%$ and 20 with tightness $80 \%$ ). The 20 instances in Set 5 has 15 variables, 10 constraints, 10 domain values (10 instances with tightness $60 \%$ and 10 instances with tightness $80 \%$ ). Table 3 gives the results.

\begin{tabular}{|c|c|c|c|c|}
\hline Set & 4 & 4 & 5 & 5 \\
\hline Tightness & $60 \%$ & $80 \%$ & $60 \%$ & $80 \%$ \\
\hline CONFLEX & 12.34 & 19.89 & 154.42 & 165.14 \\
\hline BnB & 0.44 & 0.29 & 0.05 & 0.26 \\
\hline
\end{tabular}

Table 3. Average runtime for Sets 4 and 5 (in seconds)

We experienced difficulty in running larger problems on the Windows version of the CONFLEX software. However, it is clear from these experiments that our $\mathrm{BnB}$ algorithm is considerably faster than CONFLEX.

We also solved the three sets of problems of Table 2 and a fifth set containing 50 instances, where each instance has 150 variables, 30 constraints and a domain size of 20 . All the problems in Set 5 have a maximal solution. See Table 4.

\section{Conclusion and Future Work}

We define a WS-Max-SAT problem by translating a SCSPs into propositional weighted Max-SAT problem. We present an incomplete GSAT-based algorithm to show that this translation is feasible to solve SCSPs. We also present a BnB algorithm that performs surprisingly well, outperforming the CONFLEX system.

\footnotetext{
${ }^{5}$ http://www.inra.fr/internet/Departements/MIA/T//conflex/adressesConflex.html
} 


\begin{tabular}{|c|c|c|c|c|c|c|c|}
\hline Set & 1 & 1 & 2 & 2 & 3 & 3 & 5 \\
\hline $\mathrm{T}$ & $70 \%$ & $90 \%$ & $70 \%$ & $90 \%$ & $70 \%$ & $90 \%$ & $90 \%$ \\
\hline Runtime & 0.27 & 0.48 & 0.35 & 0.52 & 0.86 & 1.52 & 5.30 \\
\hline
\end{tabular}

Table 4. Runtime for the BnB algorithm (in seconds)

A next step is to investigate the adaptation of existing efficient Weighted MaxSAT algorithms to solve WS-Max-SAT. We also plan to test our BnB algorithm by solving benchmark problems.

\section{References}

1. Bistarelli, S., Montanari, U., Rossi, F.: Semiring-based constraint solving and optimization. Journal of the ACM 44(2) (1997) 201-236

2. Wilson, M., Borning, A.: Hierarchical constraint logic programming. Journal of Logic Programming 16 (1993) 277-318

3. Dubois, D., Fargier, H., Prade, H.: The calculus of fuzzy restrictions as a basis for flexible constraint satisfaction. In: Proc. of IEEE Conf on Fuzzy Syst. (1993)

4. Fargier, H., Lang, J.: Uncertainty in constraint satisfaction problems: a probabilistic approach. In: ECSQARU-1993. (1993)

5. Georget, Y., Codognet, P.: Compiling semiring-based constraint with $\operatorname{clp}(\mathrm{FD}, \mathrm{s})$. In: CP-1998. (1998)

6. Rossi, F., Pilan, I.: Abstracting soft constraints: Some experimental results. In: Proc. of Joint Annual Workshop of ERCIM Working Group on Constraints and the CologNET area on Constraint and Logic Programming. (2003)

7. Bistarelli, S., Fargier, H., Montanari, U., Rossi, F., Schiex, T., Verfaillie, G.: Semiring-based CSPs and valued CSPs: Basic properties and comparison. Constraints 4 (1999) 199-240

8. Bistarelli, S., Fung, S., Lee, J., Leung, H.: A local search framework for semiringbased constraint satisfaction problems. In: Soft-2003. (2003)

9. Wilson, N.: Decision diagrams for the computation of semiring valuations. In: IJCAI-05. (2005)

10. Xing, Z., Zhang, W.: MaxSolver: An efficient exact algorithm for (weighted) maximum satisfiability. Artificial Intelligence 164(1-2) (2005) 47-80

11. Alsinet, T., Manya, F., Planes, J.: Improved exact solver for weighted Max-SAT. In: SAT-2005. (2005) 371-377

12. Gent, I.P.: Arc consistency in SAT. In: ECAI-2002. (2002)

13. Walsh, T.: SAT v CSP. In: CP-2000. (2000) 441-456

14. Bennaceur, H.: The satisfiability problem regarded as a constraint satisfaction problem. In: ECAI 1996. (1996) 155-159

15. Selman, B., Levesque, H., Mitchell, D.: A new method for solving hard satisfiability problems. In: AAAI-1992. (1992) 440-446

16. Li, C.M., Manyà, F., Planes, J.: Detecting disjoint inconsistent subformulas for computing lower bounds for Max-SAT. In: AAAI-06. (2006)

17. de Givry, S., Larrosa, J., Meseguer, Schiex, T.: Solving Max-SAT as weighted CSP. In: CP-2003. (2003)

18. Larossa, J., Heras, F.: Resolution in Max-SAT and its relation to local consistency in weighted CSPs. In: IJCAI-05. (2005) 